\title{
Mono-Higgs signature in fermionic dark matter model
}

\author{
Karim Ghorbani and Leila Khalkhali
}

Physics Department, Faculty of Sciences, Arak University, Arak 38156-8-8349, Iran

\begin{abstract}
In light of the Higgs boson discovery, we explore mono-Higgs signature in association with dark matter pair production at the LHC in a renormalizable model with a fermionic dark matter candidate. For two channels with $\gamma \gamma+$ MET and $b \bar{b}+$ MET in the final state we simulate the SM backgrounds and signal events at $\sqrt{s}=14$ $\mathrm{TeV}$. We then estimate the LHC sensitivities for various benchmark points for two integrated luminosities $\mathcal{L}=300 \mathrm{fb}^{-1}$ and $\mathcal{L}=3 \mathrm{ab}^{-1}$. We constrain the Yukawa coupling of the dark matter-SM interaction, taking into account bounds from monoHiggs signature, observed dark matter relic density, Higgs physics, perturbativity requirement and electroweak measurements. Concerning the mono-Higgs search, it turns out that the channel with the largest branching ratio, $b \bar{b}$ channel, provides better sensitivity. There are found regions in the parameter space of the model compatible with all the bounds mentioned above which can be reached in future LHC studies.
\end{abstract}

keywords: Dark matter theory, Collider searches, Mono-Higgs 


\section{Introduction}

It is well established that dark matter (DM) constitutes about $26 \%$ of the energy-matter content of the Universe 1, 2. The problem of dark matter which remains unanswered in the standard model (SM) of particle physics can be explained by weak-scale scenarios within the freeze-out mechanism in the early Universe [3]. Weakly interacting massive particles (WIMPs) are generically well motivated DM candidates in this mechanism, for a review see [4]. From particle physics vantage point, the main question is what would be the underlaying interactions between DM and the SM particles.

Direct detection experiments such as LUX [5] and XENON [6] are proposed to probe such probable interactions. So far in these experiments, there is found no indication of any DM elastic scattering off the target nuclei. However, the experimental results provide us with bounds on the spin-independent direct detection cross section. The upper limits on the elastic scattering cross section may constrain strongly the parameter space of theoretical models beyond the SM. It is worth noting that by appealing to some specific DM interactions with ordinary matter, the experimental bound can be evaded.

On the other hand, the discovery of the Higgs boson [7, 8] enriched the physics at the scale of electroweak symmetry breaking, i.e., at $\sim \mathcal{O}(100) \mathrm{GeV}$. Thus, one intriguing question to ask is whether the nature of dark matter is connected in some ways to this weak scale physics. The search for the underlying nature behind DM in processes in connection with the Higgs production at the LHC is a new avenue as indirect detection of DM.

One such processes which can happen at the LHC is called mono-Higgs where DM production is accompanied by a single Higgs boson in the final state, see mono-Higgs studies within both effective field theory approach and simplified models in [9-14]. Since dark matter is neutral and interacts weakly with ordinary matter, it will escape the detector recoiling against the Higgs, and leaves some amount of missing transverse energy $\left(\mathbb{E}_{T}\right.$ or MET). Recent LHC search for dark matter in association with a Higgs can be found in [15, 16].

Mono-Higgs is among a large class of processes with the production of DM in a collider in association with a visible final state $\mathrm{X}$, which is generally dubbed mono-X processes. Mono-X searches and studies are carried out for various $\mathrm{X}$, for instance as a light or heavy jet in [17 26], as a $\mathrm{Z}$ or $\mathrm{W}$ boson in [27 36], and as a photon in [37 40]. Along the same lines, works with emphasis on pseudoscalar mediator can be found in [41-45].

In this work we explore a renormalizable model with fermionic DM candidate which has a particular interaction with the SM particles. The fermionic DM is connected directly to a real pseudoscalar singlet $\phi$ through the operator $\phi \bar{\chi} \gamma^{5} \chi$. Since we would like to stick to a renormalizable model, gauge invariance allows only for interaction between the pseudoscalar singlet and the SM Higgs such as a dimension-4 operator $\phi^{2} H^{\dagger} H$.

This model has a particular characteristic where its DM candidate can escape not

only the current direct detection experiments but also the near future experiments like 
XENON1T. The reason is due to the fact that DM-nucleon elastic scattering cross section in this model is velocity suppressed [46 48]. Since direct detection experiments puts no constraints in this type of models, it is deemed interesting to investigate other avenues such as dark matter production in association with mono-Higgs at the LHC. We will study mono-Higgs signals in two important channels: $\gamma \gamma+$ MET and $b \bar{b}+$ MET in the final states. The main purpose in this study is to constrain the model parameter space at $95 \%$ confidence level (CL) in mono-Higgs searches besides constraints coming from observed DM relic density, invisible Higgs decay measurements and electroweak precision measurements.

This article has the following structure. In the next section, we introduce a simplified renormalizable dark matter model. In section 3 we discuss all possible constraints concerning our model. In section 4 we will then investigate the LHC sensitivities to monoHiggs signature at the LHC for two integrated luminosities $\mathcal{L}=300 \mathrm{fb}^{-1}$ and $\mathcal{L}=3 \mathrm{ab}^{-1}$. We conclude in section 5 ,

\section{Dark matter model}

We describe in this section a renormalizable dark matter model introducing a new Dirac field $(\chi)$ to become our DM candidate and a pseudoscalar field $(\phi)$ as a mediator, both being gauge singlet under $S U(3)_{c} \times S U(2)_{L} \times U(1)_{Y}$ [49, 50]. In this model the CPinvariant interaction Lagrangian consists of an interaction term connecting the Dirac and the pseudoscalar fields and an interaction term connecting the pseudoscalar to the SMHiggs doublet $H$, as

$$
\mathcal{L}_{\text {int }}=-i g_{\chi} \phi \bar{\chi} \gamma^{5} \chi-\lambda_{1} \phi^{2} H^{\dagger} H
$$

The SM-Higgs field gets a non-zero vacuum expectation value $v_{h}$ and we therefore parametrize the Higgs field as

$$
H=\frac{1}{\sqrt{2}}\left(\begin{array}{c}
0 \\
v_{h}+h^{\prime}
\end{array}\right)
$$

where $v_{h}=246 \mathrm{GeV}$. The pseudoscalar potential being CP-invariant is introduced by the Lagrangian

$$
\mathcal{L}_{\phi}=\frac{1}{2}\left(\partial_{\mu} \phi\right)^{2}-\frac{m^{2}}{2} \phi^{2}-\frac{\lambda}{24} \phi^{4}
$$

and the known SM-Higgs potential is given by

$$
V_{H}=\mu_{H}^{2} H^{\dagger} H+\lambda_{H}\left(H^{\dagger} H\right)^{2} .
$$

It is assumed that the pseudoscalar field acquires a non-zero vacuum expectation value, thus

$$
\phi=v_{\phi}+s .
$$


It is readily seen that the CP symmetry is no longer preserved since $v_{\phi} \neq 0$. The second term in the interaction Lagrangian, eq. (1), gives rise to a mixing term in the $h^{\prime}-s$ mass matrix for a non-zero $v_{\phi}$. The mass matrix is diagonalized by redefining the scalar fields as

$$
h=\sin \beta s+\cos \beta h^{\prime}, \quad \rho=\cos \beta s-\sin \beta h^{\prime},
$$

where the mixing angle is defined by

$$
\tan 2 \beta=\frac{2 \lambda_{1} v_{\phi} v_{h}}{\lambda_{H} v_{h}^{2}-\lambda v_{\phi}^{2} / 6} .
$$

After field redefinition, the interaction Lagrangian for $\rho$ and $h$ fields becomes

$$
\begin{array}{r}
\mathcal{L}_{\text {int }}+\mathcal{L}_{\phi}=-i g_{\chi}(h \sin \beta+\rho \cos \beta) \bar{\chi} \gamma^{5} \chi-\left(\cos ^{2} \beta \sin \beta \lambda v_{\phi}+6 \cos \beta \sin ^{2} \beta \lambda_{H} v_{h}\right. \\
\left.-6 \cos \beta \sin ^{2} \beta \lambda_{1} v_{h}+2 \cos \beta \lambda_{1} v_{h}+6 \sin ^{3} \beta \lambda_{1} v_{\phi}-4 \sin \beta \lambda_{1} v_{\phi}\right) \rho^{2} h \\
-\left(\cos \beta \sin ^{2} \beta \lambda v_{\phi}-6 \cos ^{2} \beta \sin \beta \lambda_{H} v_{h}-6 \sin ^{3} \beta \lambda_{1} v_{h}+4 \sin \beta \lambda_{1} v_{h}\right. \\
\left.-6 \cos \beta \sin ^{2} \beta \lambda_{1} v_{\phi}+2 \cos \beta \lambda_{1} v_{\phi}\right) h^{2} \rho+\ldots,
\end{array}
$$

where ellipsis indicate terms with higher number of $h$ and $\rho$ fields. Moreover, quark interaction with $\rho$ and $h$ fields becomes

$$
\mathcal{L}=-\sum_{q} \frac{m_{q}}{v_{h}} q \bar{q}(h \cos \beta-\rho \sin \beta) .
$$

There is an effective Lagrangian as an extension to the SM which gives us contact interaction between the Higgs and photons [51,52]. After field rotation the effective Lagrangian becomes

$$
\mathcal{L}_{\text {eff }}=-\frac{1}{4} g(h \cos \beta-\rho \sin \beta) F_{\mu \nu} F^{\mu \nu},
$$

with

$$
\begin{array}{r}
g=\frac{e^{2}}{4 \pi^{2} v_{h}} \frac{47}{18}\left(1+\frac{66}{235} \tau_{w}+\frac{228}{1645} \tau_{w}^{2}+\frac{696}{8225} \tau_{w}^{3}+\frac{5248}{90475} \tau_{w}^{4}\right. \\
\left.+\frac{1280}{29939} \tau_{w}^{5}+\frac{54528}{1646645} \tau_{w}^{6}-\frac{56}{705} \tau_{t}-\frac{32}{987} \tau_{t}^{2}\right)
\end{array}
$$

where $\tau_{w}=\frac{m_{h}^{2}}{4 m_{w}^{2}}$ and $\tau_{t}=\frac{m_{h}^{2}}{4 m_{t}^{2}}$. The effective coupling $g$ is obtained in the SM after integrating out top quark or $\mathrm{W}$ boson in the loops. The effective Lagrangian above is employed by the event generator to implement the Higgs decay into diphoton.

In this work we take the mixing angle as a free parameter and instead obtain the quartic couplings in terms of the mixing angle and physical masses of the scalars,

$$
\lambda_{H}=\frac{m_{\rho}^{2} \sin ^{2} \beta+m_{h}^{2} \cos ^{2} \beta}{2 v_{h}^{2}}, \quad \lambda=\frac{m_{\rho}^{2} \cos ^{2} \beta+m_{h}^{2} \sin ^{2} \beta}{v_{\phi}^{2} / 3}, \quad \lambda_{1}=\frac{m_{\rho}^{2}-m_{h}^{2}}{4 v_{h} v_{\phi}} \sin 2 \beta .
$$


We will restrict our numerical computations to regions in the parameter space that the stability of the total potential is guaranteed by satisfying the relations, $\lambda \lambda_{H}>6 \lambda_{1}^{2}$ (when $\left.\lambda_{1}<0\right), \lambda>0$ and $\lambda_{H}>0$. Moreover, the model remains perturbative when we choose $\left|\lambda_{i}\right|<4 \pi$.

\section{The constraints in the model}

In this section we introduce all the possible constraints on the DM model prior to the ones we will find from mono-Higgs searches.

\subsection{Constraints from Higgs physics and Oblique parameters}

When $m_{\chi}<m_{h} / 2$, the SM Higgs in the above mentioned model can decay invisibly into a pair of DM with decay width,

$$
\Gamma_{\mathrm{inv}}=\frac{g_{\chi}^{2} m_{h} \sin ^{2} \beta}{8 \pi}\left(1-\frac{4 m_{\chi}^{2}}{m_{h}^{2}}\right)^{1 / 2} .
$$

The CMS analysis [53] which presents a combined searches in two channels, one for a SM Higgs production via vector boson fusion and another for a Higgs production in association with a $\mathrm{Z}$ boson, imposes the strongest bound on the branching ratio of the invisibly decaying Higgs. The analysis found $\mathrm{BR}(h \rightarrow$ inv. $) \lesssim 0.58$ at $2 \sigma$ level. In addition, bounds on invisible decays of the Higgs boson from Higgs production in association with top quarks finds $\mathrm{BR}(h \rightarrow$ inv. $) \lesssim 0.24$ [54]. Applying this latter bound we find an upper limit for the combination of the Higgs-DM coupling and the mixing angle,

$$
\left|g_{\chi} \tan \beta\right|<\frac{5.05(\mathrm{MeV})^{1 / 2}}{\left(m_{h}^{2}-4 m_{\chi}^{2}\right)^{1 / 4}}
$$

where we used the Standard Model prediction for the Higgs total decay width, $\Gamma_{\mathrm{h}}^{\mathrm{SM}}=4$ $\mathrm{MeV}$ [55].

It is possible to constrain the single parameter, $\beta$, by applying the recent measurements on the Higgs production and its decay into SM final states [56]. The quantity which is measured by CMS and ATLAS is called signal strength, $\mu$, and is defined as

$$
\mu_{i}^{f}=\frac{\left(\sigma_{i} \times \mathrm{BR}^{f}\right)_{\mathrm{Exp}}}{\left(\sigma_{i} \times \mathrm{BR}^{f}\right)_{\mathrm{SM}}} .
$$

The SM Higgs production via channel $i$ is denoted by $\sigma_{i}$ and $\mathrm{BR}^{f}$ is the branching ratio of Higgs decaying into SM final state $f$. The combined result obtained by a fit over all various production and decay channels reads $\mu=1.09 \pm 0.1$. Since in our model there is mixing between the pseudoscalar and the Higgs, $\sigma_{i}$ is scaled by a factor $\cos ^{2} \beta$. If we 
assume that $\Gamma_{\mathrm{Higgs}}^{\mathrm{inv}}<<\Gamma_{\mathrm{Higgs}}^{\mathrm{SM}}$, then $\mathrm{BR}^{f}$ remains the same as its $\mathrm{SM}$ value. Therefore in this work $\mu \sim \cos ^{2} \beta$. The experimental finding will then restrict the mixing angle $\beta$ to values smaller than $\sim 0.1$ at $1 \sigma$ precision.

If we consider mixing angles smaller than $\sim 0.1$, then constraints from oblique parameters $S$ and $U$ are negligible. When we add an additional (pseudo)scalar to the SM, the oblique parameter $T$ will be modified as follows [57],

$$
\begin{aligned}
T^{B S M}=-\left(\frac{3}{16 \pi s_{w}^{2}}\right)\{ & \cos ^{2} \beta\left[\frac{1}{c_{w}^{2}}\left(\frac{m_{h}^{2}}{m_{h}^{2}-m_{Z}^{2}}\right) \ln \frac{m_{h}^{2}}{m_{Z}^{2}}-\left(\frac{m_{h}^{2}}{m_{h}^{2}-m_{W}^{2}}\right) \ln \frac{m_{h}^{2}}{m_{W}^{2}}\right] \\
& \left.+\sin ^{2} \beta\left[\frac{1}{c_{w}^{2}}\left(\frac{m_{\rho}^{2}}{m_{\rho}^{2}-m_{Z}^{2}}\right) \ln \frac{m_{\rho}^{2}}{m_{Z}^{2}}-\left(\frac{m_{\rho}^{2}}{m_{\rho}^{2}-m_{W}^{2}}\right) \ln \frac{m_{\rho}^{2}}{m_{W}^{2}}\right]\right\},
\end{aligned}
$$

in which $c_{w}=\cos \theta_{W}$. For $\beta=0$, we have $T^{B S M}=T^{S M}$. The best fit on electroweak measurements dictates $\Delta T=0.01 \pm 0.12$ [58], where $\Delta T=T^{B S M}-T^{S M}$. The mixing angle is constrained insignificantly if we take $\beta$ less than $\sim 0.1$.

\subsection{DM relic density}

Furthermore, the combined results from Planck and WMAP provide us with the DM relic density, $0.1172<\Omega_{\mathrm{DM}} \mathrm{h}^{2}<0.1226$. This observation will exclude some regions in the model parameter space. In order to find the present amount of DM number density and then the DM relic density for various models, it requires the numerical solution of the Boltzmann equation at the freeze-out condition,

$$
\frac{d n}{d t}+3 H n=-\left\langle\sigma_{\mathrm{ann}} v_{\mathrm{rel}}\right\rangle\left[n^{2}-\left(n^{\mathrm{eq}}\right)^{2}\right] .
$$

Here $\left\langle\sigma_{\text {ann }} v_{\text {rel }}\right\rangle$ is the thermal average of DM annihilation cross sections times the relative velocity and $n^{\text {eq }}$ is the total number of $\chi$ particles at equilibrium.

In this work, to obtain the DM relic density we make use of the numerical package microOMEGAs [59] which exploits the package CalcHEP [60] to compute the relevant annihilation cross sections. One may consult [50] for some detailed DM phenomenology of the model under discussion in the present work.

\section{Mono-Higgs signature at the LHC}

We devote this section to study the LHC sensitivity to mono-Higgs plus MET production at $\sqrt{s}=14 \mathrm{TeV}$ for two prospective integrated luminosities, $\mathcal{L}=300 \mathrm{fb}^{-1}$ and $\mathcal{L}=$ $3 \mathrm{ab}^{-1}$. We generate our signal and background unweighted events at leading order in MadGraph5aMC@NLO v2.1.2 [61,62] with the CTEQ6L1 PDF [63] which are then passed on to PYTHIA6 64] for patron showering and hadronization. For detector simulation we 

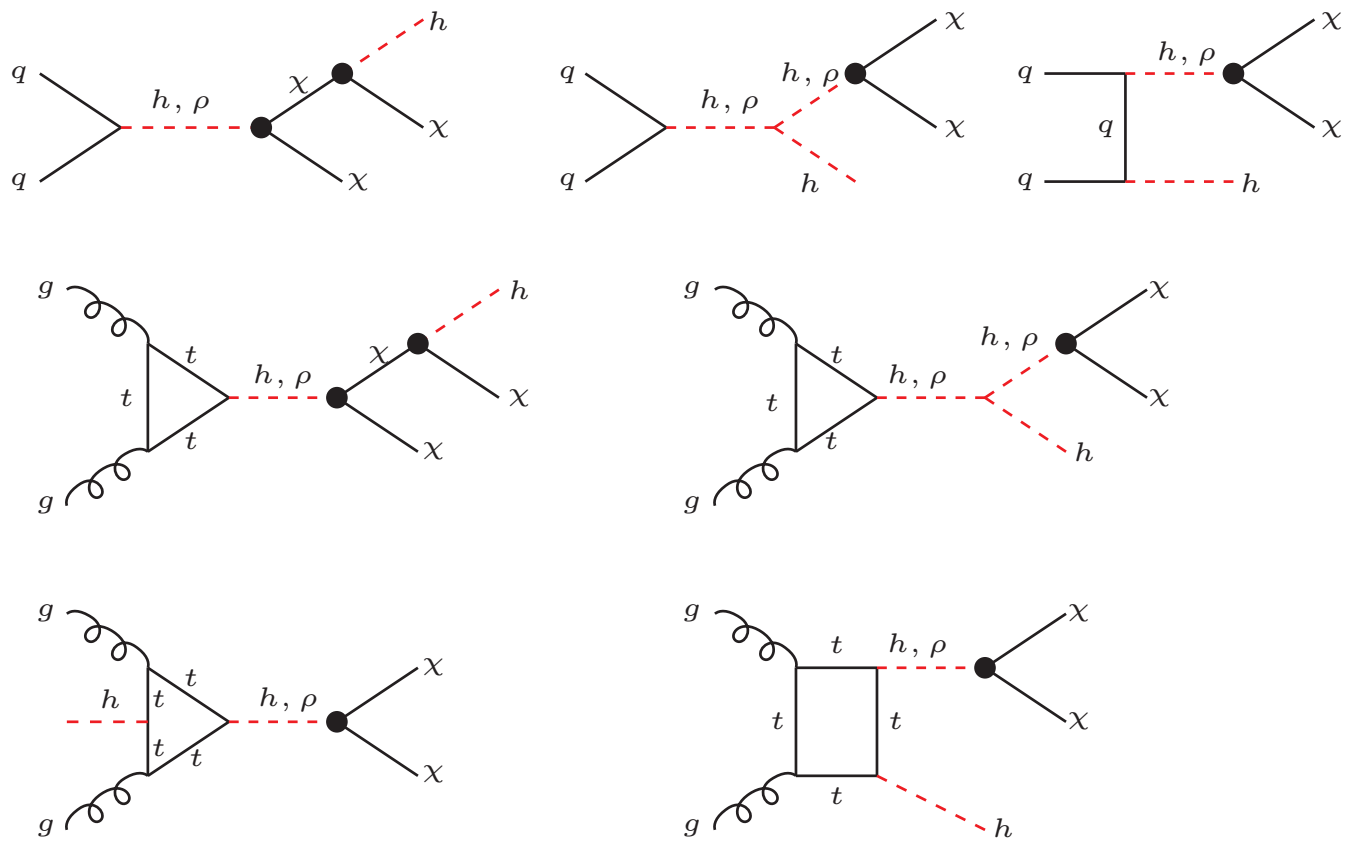

Figure 1: Feynman diagrams relevant for the mono-Higgs production at the LHC in the process $p p \rightarrow \chi \chi h$.

employ Delphes 3 [65] (to simulate CMS detector) which integrates Fastjet [66] with $R=0.5$ to allow jet reconstruction using the anti- $k_{t}$ algorithm [67] as jet clustering algorithm. We employ MadAnalysis 5 [68,69] to perform the analysis in this work.

In this study we take the signal production cross section at leading order but the production cross sections for various background samples will be corrected by using appropriate $k$-factors so as to normalize them to higher order calculations. Therefore, our signal efficiencies in this work are somewhat underestimated.

For the signal process with missing transverse energy plus Higgs in the final state, $p p \rightarrow \chi \chi h$, we show the relevant Feynman diagrams in Fig. 1. This process is induced through quark or gluon fusions in $p p$ collisions via s-, t- and u-channels. In our numerical investigation we find out that diagrams with gluon fusion have dominant contributions to the total cross section. Our numerical results for the signal production cross section for various benchmark points at $\sqrt{s}=14 \mathrm{TeV}$ are presented as a function of DM mass in Fig. 2 for $\sin \beta=0.1$ and $\sin \beta=0.01$. The results shown for three mediator masses $m_{\rho}=100,400$ and $800 \mathrm{GeV}$, indicate strong dependency of the signal production cross section on the mediator mass when $m_{\mathrm{DM}}>m_{h} / 2$. In the region with $m_{\mathrm{DM}}<m_{h} / 2$, since the Higgs resonance is accessible it dominates the cross section. In this region the signal cross sections remain almost steady and then drop off. The same reasoning can be applied for the $\rho$ resonance. We have checked that the signal cross section does not 

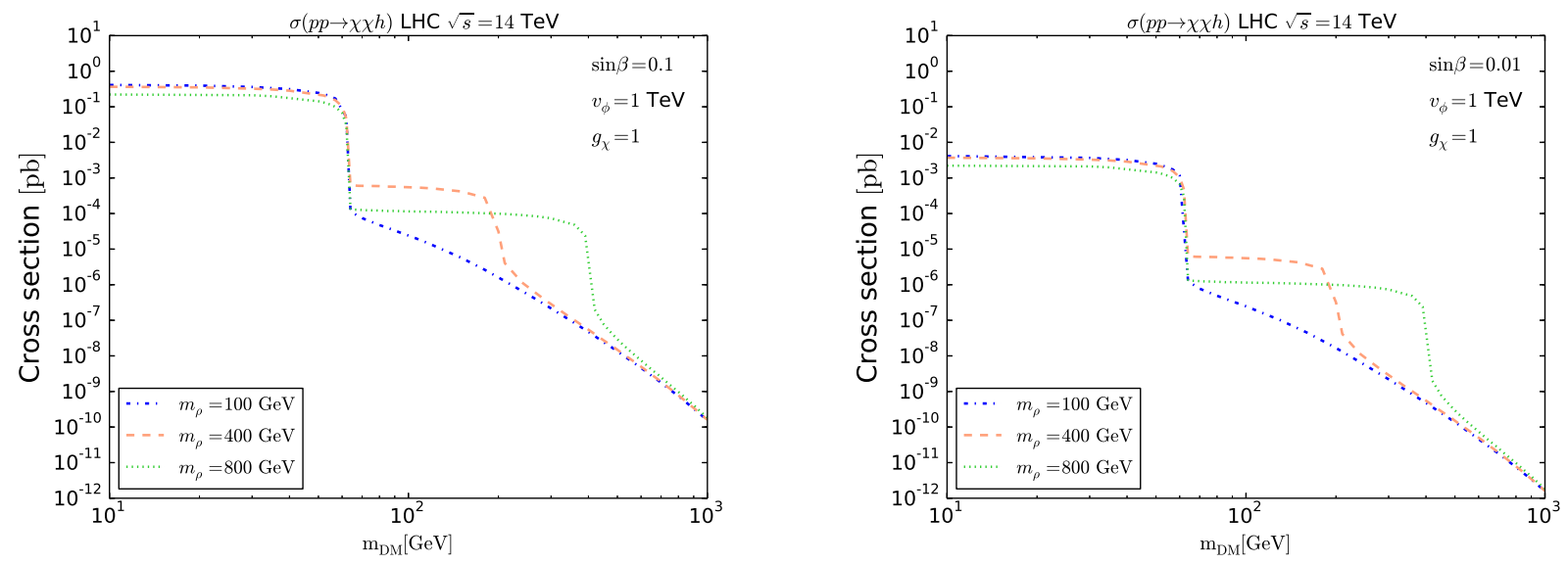

Figure 2: Mono-Higgs production cross section via the process $p p \rightarrow \chi \chi h$ for various benchmark models at $\sqrt{s}=14 \mathrm{TeV}$ for $v_{\phi}=1 \mathrm{TeV}, \sin \beta=0.1$ (left) and $\sin \beta=0.01$ (right).

change significantly by going from $v_{\phi}=0.5 \mathrm{TeV}$ to $v_{\phi}=1 \mathrm{TeV}$. Moreover, the signal production cross section is proportional to $g_{\chi}^{2}$ based on the Feynman diagrams in Fig. 1. We choose $v_{\phi}=1 \mathrm{TeV}, g_{\chi}=1$ and $\sin \beta=0.1$ throughout our computations.

Our focus in this research is to estimate the LHC sensitivity reach in two important Higgs decay channels: $h \rightarrow \gamma \gamma$ and $h \rightarrow b \bar{b}$.

\subsection{Two photon channel}

This decay mode is important because we deal with small background events which make a clean environment for experimental measurements. However, the Higgs decay into two photons has a small branching ratio, $\operatorname{Br}(h \rightarrow \gamma \gamma)=2.28 \times 10^{-3}$, and hence the signal production rate will be comparatively low.

Important backgrounds to the final state $\gamma \gamma+\mathrm{MET}$ are as we list:

1. $Z h$ with $h \rightarrow \gamma \gamma$ and $\left(Z \rightarrow \nu \bar{\nu}\right.$ or $Z \rightarrow \tau^{+} \tau^{-}$where $\tau$ decay produces some missing energy). This is an irreducible background and is denoted by $\mathrm{VH}$ in plots.

2. Wh with $h \rightarrow \gamma \gamma$ and $W \rightarrow l \nu$, denoted by $\mathrm{VH}$.

3. $Z \gamma \gamma$ with $Z \rightarrow \nu \nu$ or $Z \rightarrow \tau^{+} \tau^{-}$(where $\tau$ decay produces some missing energy), denoted by VAA.

4. $W \gamma \gamma$ with $W \rightarrow l \nu$, denoted by VAA.

5. $\gamma \gamma$ through Higgs production or via non-resonant production, denoted by $\mathrm{H}$ and AA respectively. 

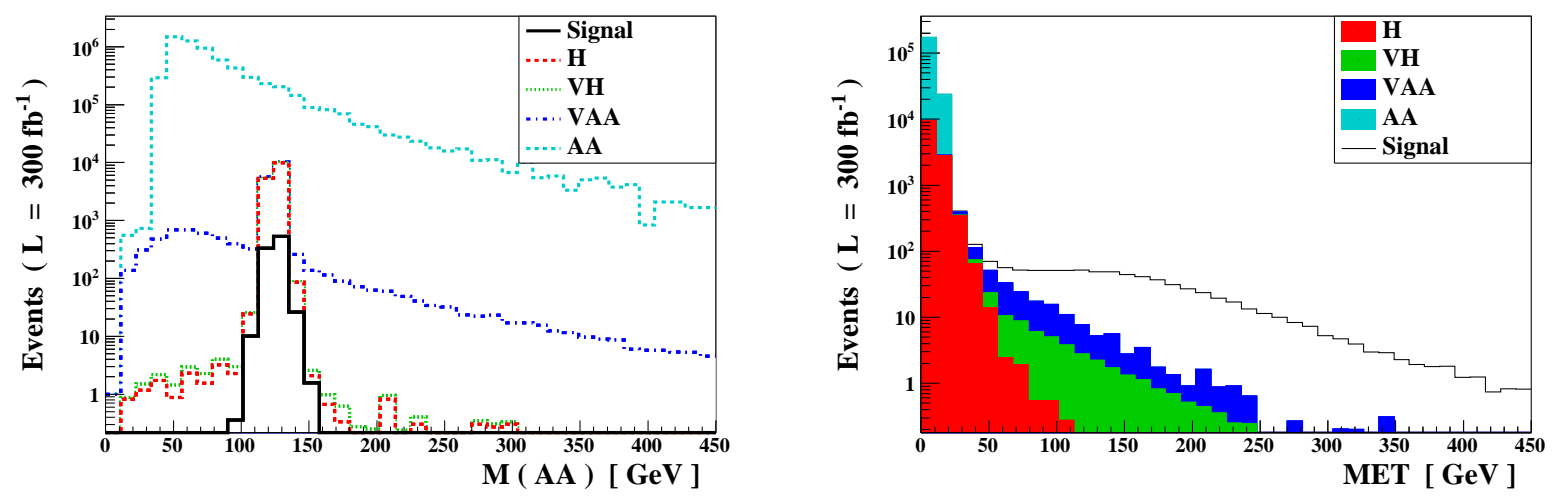

Figure 3: For $\gamma \gamma+$ MET channel at $\sqrt{s}=14 \mathrm{TeV}$ with $\mathcal{L}=300 \mathrm{fb}^{-1}$, in the left panel is the diphoton invariant mass for the signal and background processes and in the right panel is distributions of the missing transverse energy for the signal and backgrounds after applying the event selections discussed in the text. Signal is normalized to a nominal cross section of $5 \mathrm{fb}$.

We consider as event selections, the existence of two photons in the final state with transverse momentum $p_{T}>20 \mathrm{GeV}$ and rapidity $|\mu|<2.5$ and we veto electrons or muons with $p_{T}>20 \mathrm{GeV}$ and $|\mu|<2.5$. In order to improve signal to background efficiency we impose the cut $120 \mathrm{GeV}<m_{\gamma \gamma}<130 \mathrm{GeV}$ for invariant mass of diphoton.

In Fig. 3 we present the diphoton invariant mass after the event selections are applied and also distributions for signal and background events after both the event selections and the cut are taken into account. The SM backgrounds are estimated using $k$-factors to normalize the leading order (LO) cross sections to their values at NLO or beyond. We use $k=1.65$ [70] for $Z \gamma \gamma$ and the same value for $W \gamma \gamma, k=1.3$ [71] for $Z h$ and the same value for $W h, k=1.8$ [72] for Higgs production which includes NNLO QCD and NLO EW corrections and $k=1.6$ [73] for $\gamma \gamma$ production.

We find that the cut MET $>150 \mathrm{GeV}$ on missing transverse energy together with the cut on $m_{\gamma \gamma}$ give the maximum sensitivity to the signal. The former cut reduces background events with MET stemming from mismeasurement of identified physical objects like photons or soft radiations in the background processes denoted by AA or $\mathrm{H}$. We show in Table. 1 the expected signal and background events at $\sqrt{s}=14 \mathrm{TeV}$ with $\mathcal{L}=300 \mathrm{fb}^{-1}$ for a signal benchmark with $m_{\mathrm{DM}}=40 \mathrm{GeV}$ and $m_{\rho}=100 \mathrm{GeV}$. According to the results given in Table. 1 backgrounds coming from $Z / W \gamma \gamma$ and $Z / W h$ are the dominant ones.

\subsection{Two b-jet channel}

Even though our signal with Higgs decaying into two b-quarks is associated with quite large SM backgrounds, this decay mode has the largest branching ratio, $\operatorname{Br}(h \rightarrow b \bar{b})=$ 0.577. So it would be interesting to see if this channel can give a sensible sensitivity reach 


\begin{tabular}{ll}
\hline \hline$p p \rightarrow \gamma \gamma+\mathbb{E}_{T}$ & $\mathbb{E}_{T}>150 \mathrm{GeV}$ \\
\hline \hline$Z \gamma \gamma+W^{ \pm} \gamma \gamma$ & $8.84 \pm 2.90$ \\
$Z h+W^{ \pm} h$ & $6.91 \pm 2.56$ \\
$h$ & $0 \pm 0$ \\
$\gamma \gamma$ & $0 \pm 0$ \\
Total backgrounds & $15.75 \pm 3.9$ \\
\hline Signal & $297.8 \pm 15.4$ \\
\hline \hline
\end{tabular}

Table 1: Signal and backgrounds for $\gamma \gamma+$ MET channel are shown with $\mathcal{L}=300 \mathrm{fb}^{-1}$ at $\sqrt{s}=14 \mathrm{TeV}$, after the event selections and cuts discussed in the text are applied. The signal events are for normalized cross section $\sigma=5 \mathrm{fb}$, and for $m_{\mathrm{DM}}=40 \mathrm{GeV}$ and $m_{\rho}=$ $100 \mathrm{GeV}$.

at the LHC. In this study we simulate only the dominant backgrounds. We have ignored backgrounds with two bosons in the final state which have negligible contributions to the total background. By choosing a suitable cut for missing transverse energy, the QCD multi-jet backgrounds can be suppressed and we therefore do not take them into account in our simulations.

We list here the most important backgrounds to the final state $b \bar{b}+$ MET as the following:

1. $Z h$ with $h \rightarrow b \bar{b}$ and $\left(Z \rightarrow \nu \bar{\nu}\right.$ or $Z \rightarrow \tau^{+} \tau^{-}$where $\tau$ decay produces some missing energy). This is an irreducible background and is denoted by $\mathrm{CH}$ in plots.

2. $W h$ with $h \rightarrow b \bar{b}$ and $W \rightarrow l \nu$, denoted by $\mathrm{CH}$.

3. $Z b \bar{b}$ with $Z \rightarrow \nu \nu$ or $Z \rightarrow \tau^{+} \tau^{-}$(where $\tau$ decay produces some missing energy), denoted by Ebb.

4. $W b \bar{b}$ with $W \rightarrow l \nu$, denoted by Ebb.

5. Higgs production with $h \rightarrow b \bar{b}$, denoted by $\mathrm{H}$.

6. $t \bar{t}$ with $t \rightarrow b l^{+} \nu_{l}$ and $\bar{t} \rightarrow \bar{b} l^{-} \bar{\nu}_{l}$, plus events in which one of the top quarks decay hadronically, denoted by TT.

Only events with two b-tagged jets with $p_{T}>50$ will be kept in our event selection. Concerning charged leptons we select to veto those with transverse momentum $p_{T}>$ 20 and rapidity $|\mu|<2.5$. The signal region is defined with the invariant mass of bquarks satisfying the cut $90 \mathrm{GeV}<m_{b b}<140 \mathrm{GeV}$. This cut will reduce significantly backgrounds with two non-resonant b-quarks, like in top pair production case, $t \bar{t}$. Figure 4 shows $b \bar{b}$ invariant mass distribution and, signal and background events with signal normalized to $1 \mathrm{pb}$ at $\sqrt{s}=14 \mathrm{TeV}$ and $\mathcal{L}=300 \mathrm{fb}^{-1}$. We find that selecting a cut 

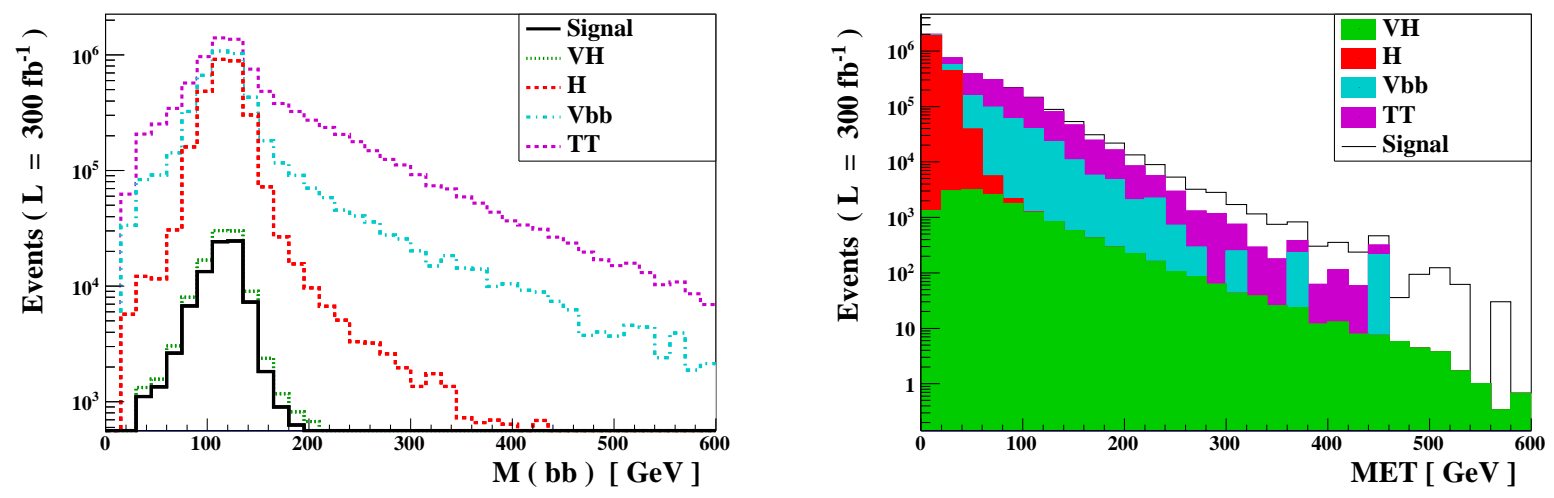

Figure 4: For $b \bar{b}+$ MET channel at $\sqrt{s}=14 \mathrm{TeV}$ with $\mathcal{L}=300 \mathrm{fb}^{-1}$, in the left panel is the $b \bar{b}$ invariant mass for the signal and background processes and in the right panel is distributions of the missing transverse energy for the signal and backgrounds after applying the event selections discussed in the text. Signal is normalized to a nominal cross section of $1 \mathrm{pb}$.

as MET $>320 \mathrm{GeV}$ will optimize the signal to background ratio. As we said earlier, background cross sections are calculated at leading order but they are scaled so as to incorporate higher order corrections.

The cross section for $Z / W+b \bar{b}$ is corrected by a factor $k=1.48$ [74]. For $Z / W+h$ cross section and $h$ cross section, $k=1.18$ [61] and $k=2$ [61] are used respectively. The LO cross section for $t \bar{t}$ production is corrected by $k=1.47$ 61]. In Table. 2 we show signal and background events for $h \rightarrow b \bar{b}$ channel for the event selections and cuts described above. In this channel even after the cuts, the remaining backgrounds are sizable.

\begin{tabular}{ll}
\hline \hline$p p \rightarrow b b+\mathbb{E}_{T}$ & $\mathbb{E}_{T}>320 \mathrm{GeV}$ \\
\hline \hline$Z b b+W^{ \pm} b b$ & $411.4 \pm 20.3$ \\
$Z h+W^{ \pm} h$ & $146 \pm 12.1$ \\
$h$ & $0 \pm 0$ \\
$t \bar{t}$ & $860.6 \pm 29.3$ \\
Total backgrounds & $1418.1 \pm 37.7$ \\
\hline Signal & $3120 \pm 55.6$ \\
\hline \hline
\end{tabular}

Table 2: Signal and backgrounds for $b \bar{b}+$ MET channel are shown with $\mathcal{L}=300 \mathrm{fb}^{-1}$ at $\sqrt{s}=14 \mathrm{TeV}$, after the event selections and cuts discussed in the text are applied. The signal events are for normalized cross section $\sigma=1 \mathrm{pb}$, and for $m_{\mathrm{DM}}=40 \mathrm{GeV}$ and $m_{\rho}=$ $100 \mathrm{GeV}$. 

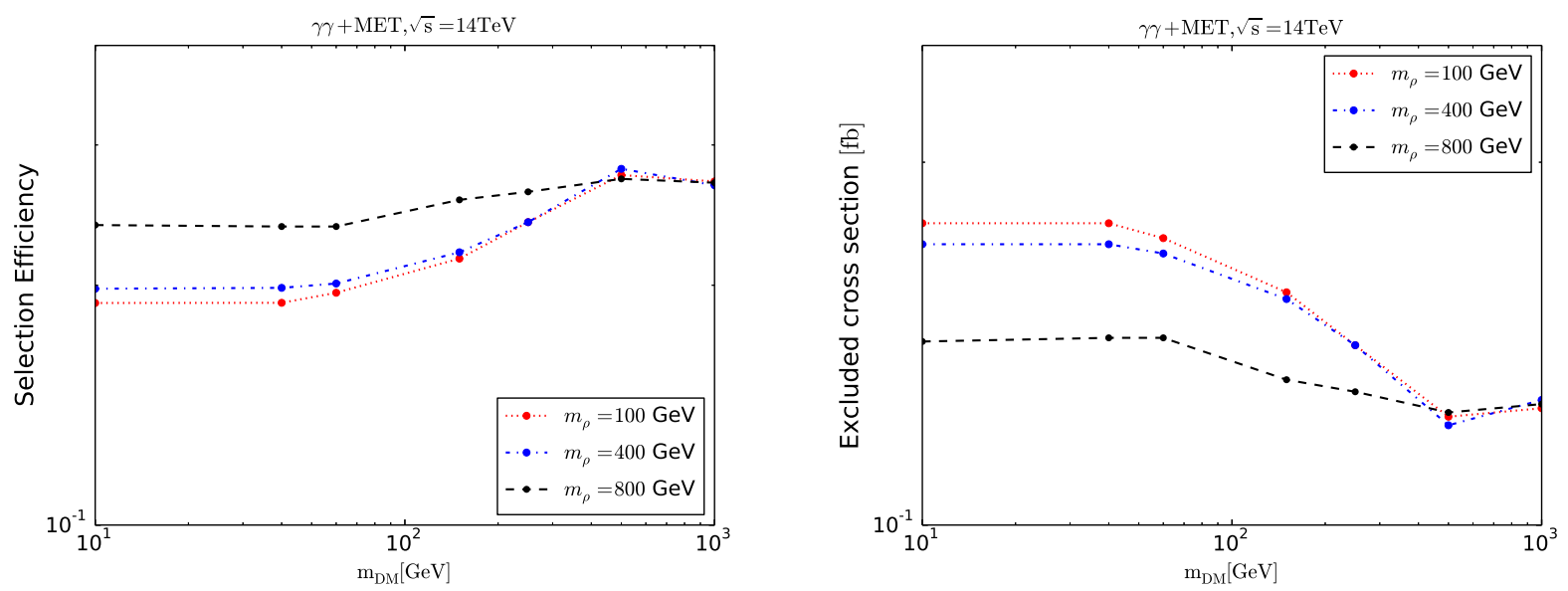

Figure 5: Shown are the selection efficiency in the $\gamma \gamma+$ MET channel (left panel) and upper limit on the cross section $\sigma(p p \rightarrow \gamma \gamma \chi \bar{\chi})$ for $\sqrt{s}=14 \mathrm{TeV}$ and $\mathcal{L}=300 \mathrm{fb}^{-1}$ at the LHC.

\subsection{Selection efficiencies and exclusions}

In this section we present our main results for the efficiencies, upper bounds on the signal cross section and upper bounds on the Yukawa coupling $g_{\chi}$ (coupling between DM and SM Higgs) for two channels $h \rightarrow \gamma \gamma$ and $h \rightarrow b \bar{b}$ based on the cuts discussed above. To find the 95\% CL exclusion for our signal benchmark points neglecting systematic uncertainties, we define significance as $\mathbb{S}=S / \sqrt{S+B}$, for signal events $S$ and total background $B$. When no signal is observed, the upper limit on the cross section (or excluded cross section) for a given signal benchmark point is obtained by requiring a significant of $\sim 2 \sigma$.

We first obtain the selection efficiencies as a function of DM mass for various benchmark points and then we evaluate the upper bound on the cross section respecting the criterion $\mathbb{S} \sim 2 \sigma$. Our result for the channel with $\gamma \gamma+$ MET in the final state is shown in Fig. 5 for the selection efficiency, $\epsilon_{s}$, (left panel) and for the excluded cross section, $\sigma_{s}$, (right panel) at $\sqrt{s}=14 \mathrm{TeV}$ and $\mathcal{L}=300 \mathrm{fb}^{-1}$. The excluded cross section is obtained by setting $S=\sigma_{s} \epsilon_{s} \mathcal{L}$ in the relation for the significance $\mathbb{S}$ and solving the equation for $\sigma_{s}$. It can be seen from the solution for $\sigma_{s}$ that the excluded cross section decreases by increasing the selection efficiency. Figure 6 shows results for the $b \bar{b}+$ MET channel. In both channels, as expected, better efficiency is achieved for larger DM mass independent of the value for $m_{\rho}$. Moreover, the efficiency gets improved for larger $m_{\rho}$. We also see that due to larger backgrounds, upper bound on the cross section $\sigma(p p \rightarrow b \bar{b} \chi \chi)$ is weaker than the one on the cross section $\sigma(p p \rightarrow \gamma \gamma \chi \chi)$.

Next, we move on to evaluate the upper limit on the Yukawa coupling. We obtain the upper limit for fixed mixing angle at $\sin \beta=0.1$. We remind that the signal production cross section is proportional to $g_{\chi}^{2}$ so that the results in Fig 2 along with the upper bounds on $\sigma(p p \rightarrow \gamma \gamma \chi \chi)$ and $\sigma(p p \rightarrow b b \chi \chi)$ provided by Fig. [5and Fig. [6 can be used to achieve 

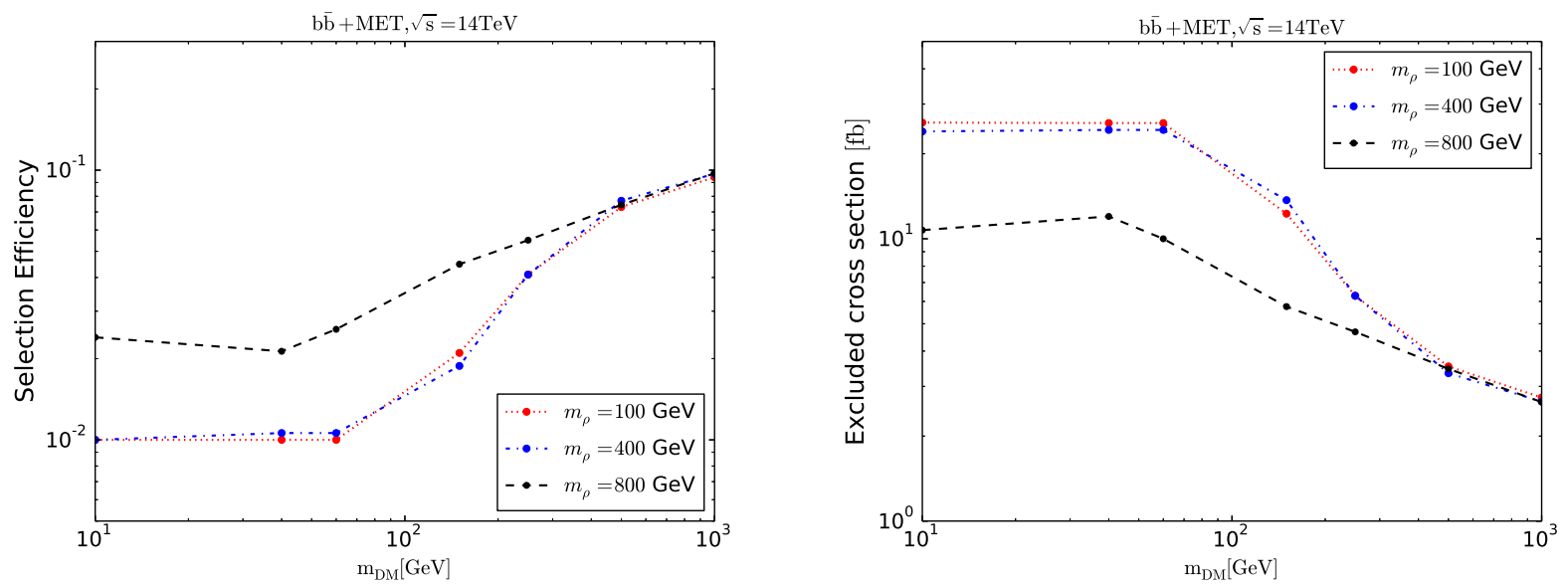

Figure 6: Shown are the selection efficiency in the $b \bar{b}+$ MET channel (left panel) and upper limit on the cross section $\sigma(p p \rightarrow b \bar{b} \chi \bar{\chi})$ for $\sqrt{s}=14 \mathrm{TeV}$ and $\mathcal{L}=300 \mathrm{fb}^{-1}$ at the LHC.

the upper limit on $g_{\chi}$ for different benchmark points.

Given our projected mono-Higgs sensitivities, in Fig. 7 we show contours which indicate the $14 \mathrm{TeV}$ LHC upper bounds on the Yukawa coupling, $g_{\chi}$, from mono-Higgs searches with $\gamma \gamma+$ MET final states. The contours are obtained for two prospective integrated luminosities $\mathcal{L}=300 \mathrm{fb}^{-1}$ and $\mathcal{L}=3 \mathrm{ab}^{-1}$ as a function of DM mass and the coupling. In addition, for each benchmark point we show the viable region in the $m_{\chi}-g_{\chi}$ plane respecting the observed DM relic density $\left(\Omega_{\chi}=\Omega_{\mathrm{DM}}\right)$ and also the viable regions with $\Omega_{\chi}=0.1 \Omega_{\mathrm{DM}}$ for three pseudoscalar masses $m_{\rho}=100,400$ and $800 \mathrm{GeV}$. It is evident in the plots that the viable value for $g_{\chi}$ drops at $m_{\mathrm{DM}} \sim m_{\rho} / 2$ and $m_{\mathrm{DM}} \sim m_{h} / 2$. The reason hinges in the fact the annihilation cross section peaks when the mediator mass is about twice the DM mass which is called the resonance region. Since the annihilation cross section is proportional to $g_{\chi}^{2}$, in order to get the observed relic density a smaller value for $g_{\chi}^{2}$ is picked up.

In the plane $m_{\chi}-g_{\chi}$, for $m_{\mathrm{DM}}<m_{h} / 2$ there is already strong constraint from invisible Higgs decay measurements which is slightly stronger than the upper limits from the mono-Higgs searches for the integrated luminosity $\mathcal{L}=300 \mathrm{fb}^{-1}$. However, at the larger luminosity the bounds from the two constraints are comparable.

For $m_{\mathrm{DM}}>m_{h} / 2$, the mono-Higgs sensitivities are higher for the intermediate mediator mass, $m_{\rho}=400 \mathrm{GeV}$. For the case with $\Omega_{\chi}=0.1 \Omega_{\mathrm{DM}}$ and $m_{\rho}=400 \mathrm{GeV}$, we find that the respective contour excludes DM masses smaller than $\sim 90 \mathrm{GeV}$ and $\sim 100 \mathrm{GeV}$ at $\mathcal{L}=300 \mathrm{fb}^{-1}$ and $\mathcal{L}=3 \mathrm{ab}^{-1}$ respectively. We note that perturbativity condition excludes regions with $g_{\chi}>4 \pi$.

We then continue our analysis for the $b \bar{b}+$ MET channel. Our results are shown for two integrated luminosities in Fig. 8 when fermionic DM constitutes fully the observed 
DM relic density or when it only makes up $10 \%$ of the observed relic density. The upshot is that in this model, the mono-Higgs searches with $b \bar{b}+$ MET in the final state has a stronger exclusion power compared with the $\gamma \gamma+$ MET channel for the Yukawa coupling. It is clearly seen in Fig. 8 that the mono-Higgs constraints get stronger with the mass of the mediator, $m_{\rho}$. The strongest constraint belongs to the case in which $\Omega_{\chi}=0.1 \Omega_{\mathrm{M}}$, $\mathcal{L}=3 \mathrm{ab}^{-1}$ and $m_{\rho}=800 \mathrm{GeV}$, where regions with $m_{\mathrm{DM}} \lesssim 120 \mathrm{GeV}$ are excluded.

There are other LHC searches that can potentially constrain the model parameter space, e.g., the pseudoscalar mass. In these searches, upper limits are found for the cross section in the processes $p p \rightarrow \rho \rightarrow\left(W^{+} W^{-}, Z Z\right)$ [75 77] and $p p \rightarrow \rho \rightarrow h h$ [78,79]. In our model, vector boson production cross section is suppressed by a factor $\sin ^{4} \beta$ and the diHiggs production cross section is suppressed by a factor $\sin ^{2} \beta$. Therefore one expects that the cross sections reside below the current upper limits for a wide range of the pseudoscalar mass. Recently, in a study [80] within the same fermionic DM model, it is confirmed that these searches put no constraints on the pseudoscalar mass. In addition, these searches cannot constrain the coupling $g_{\chi}$, since in the cross section $\sigma(p p \rightarrow$ diboson $) \sim \sigma(p p \rightarrow$ $\rho) \times \operatorname{Br}(\rho \rightarrow$ diboson $)$, the coupling $g_{\chi}$ appears only in numerator of $B r$ and plays an insignificant role.

\section{Conclusions}

We considered a model with a fermionic DM candidate which interacts with the SM Higgs due to a mixing between a pseudoscalar singlet field and the Higgs field. The specific nature of the DM-SM Yukawa interaction leads to a velocity suppressed elastic scattering cross section of DM-nucleon. Therefore near future direct detection experiments would not be able to probe the Yukawa interaction. In the model that assumes DM and the Higgs to have direct interaction, it seems plausible to search for DM signature at a collider like the LHC which is a Higgs production machine.

We have studied in this work, processes with missing energy coming from DM production associated with a Higgs boson. Our analysis are done for two channels with $\gamma \gamma+$ MET and $b \bar{b}+$ MET in the final states. We simulated the SM background processes and signal events for various benchmark points in both channels at $\sqrt{s}=14 \mathrm{TeV}$ with $\mathcal{L}=300 \mathrm{fb}^{-1}$. After event selections, backgrounds for the $\gamma \gamma+$ MET channel are found to be quite small while this is not the case for $b \bar{b}+$ MET channel.

The selection efficiencies, upper bounds on the signal cross section and upper limits on the Yukawa coupling, $g_{\chi}$, are estimated for both channels. Our main results along with constraints from invisible Higgs decay width measurements, observed DM relic density, perturbativity requirement and electroweak measurements are collected in Fig. 7 and Fig. 8 ,

It turned out that the $b \bar{b}+$ MET channel, having the largest branching ratio, gives better LHC sensitivity reach and therefore constrains the Yukawa coupling more strongly. 

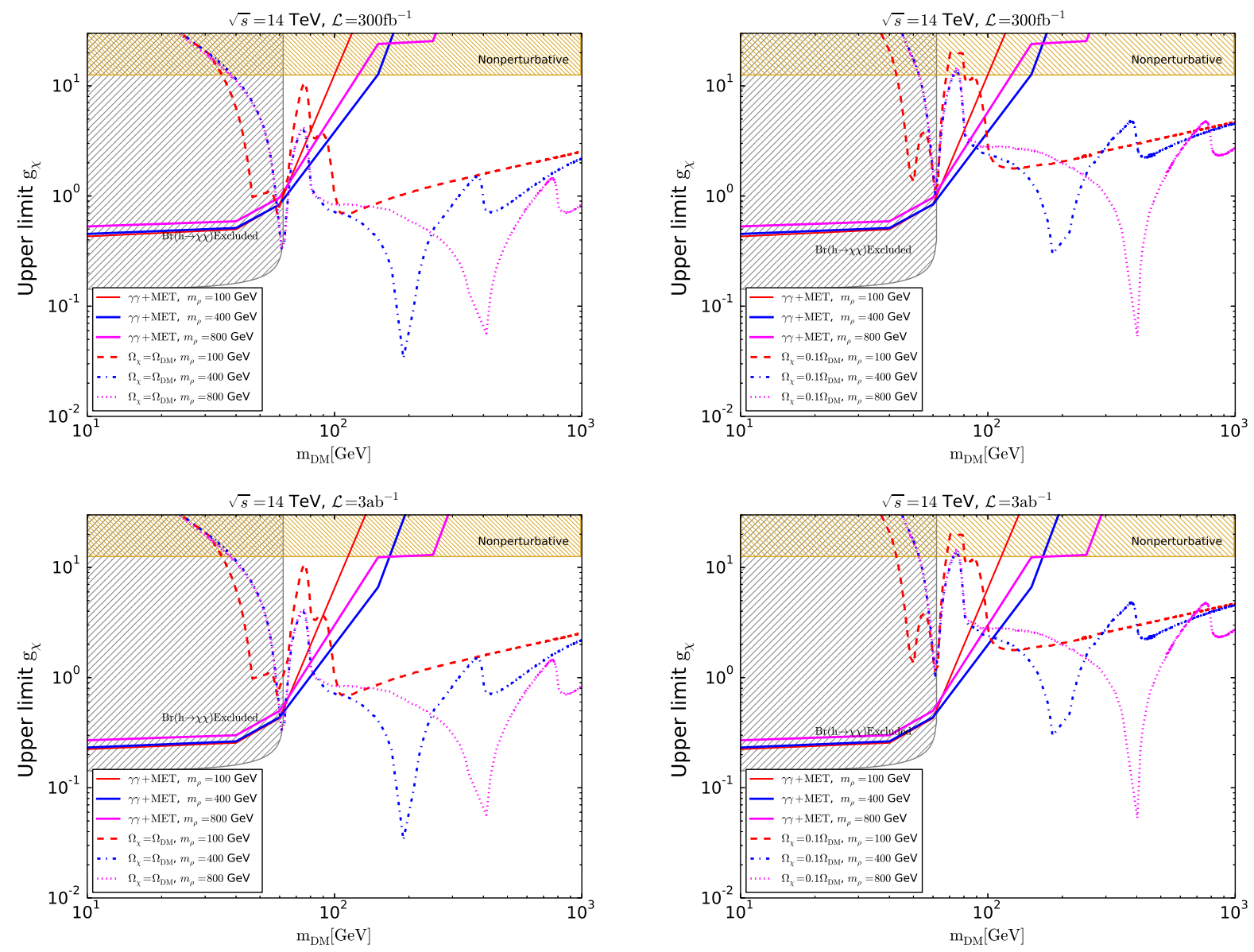

Figure 7: Shown are the projected LHC mono-Higgs sensitivities at $\sqrt{s}=14 \mathrm{TeV}$ in the $\gamma \gamma+$ MET final states with $\mathcal{L}=300 \mathrm{fb}^{-1}$ for plots on the top and with $\mathcal{L}=3 \mathrm{ab}^{-1}$ for plots on the bottom. All solid lines are contours (corresponding to 95\% CL upper limit) which exclude larger coupling, and broken lines show viable points in the $m_{\chi}-g_{\chi}$ plane for various $m_{\rho}$. Comparison made between plots on the right and plots on the left for two cases, when $\Omega_{\chi}=\Omega_{\mathrm{DM}}$ or $\Omega_{\chi}=0.1 \Omega_{\mathrm{DM}}$. The horizontal shaded area is excluded because it violates perturbativity. The shaded region on the left is excluded by the invisible Higgs decay width measurements. 

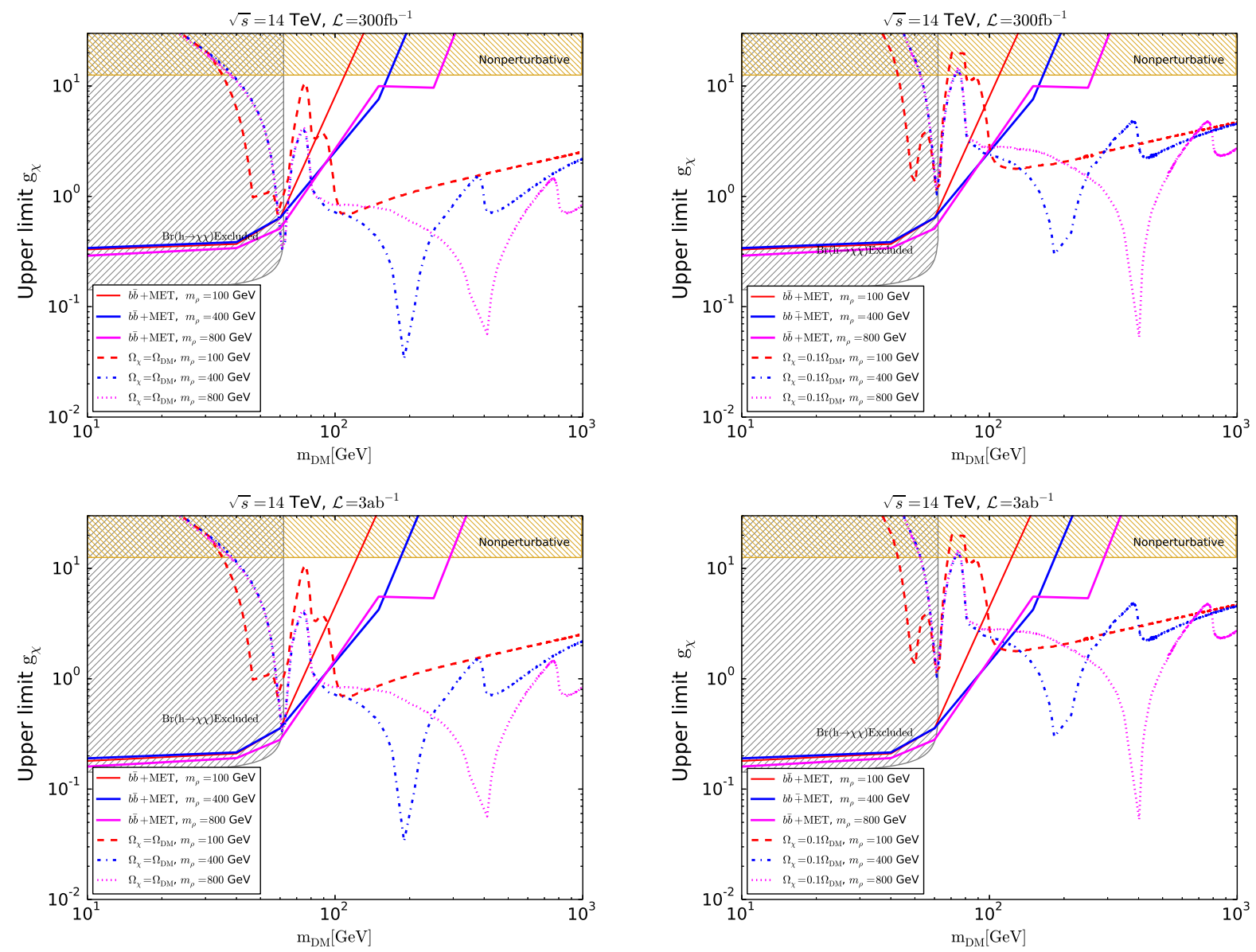

Figure 8: Shown are the projected LHC mono-Higgs sensitivities at $\sqrt{s}=14 \mathrm{TeV}$ in the $b \bar{b}+$ MET final states with $\mathcal{L}=300 \mathrm{fb}^{-1}$ for plots on the top and with $\mathcal{L}=3 \mathrm{ab}^{-1}$ for plots on the bottom. All solid lines are contours (corresponding to 95\% CL upper limit) which exclude larger coupling, and broken lines show viable points in the $m_{\chi}-g_{\chi}$ plane for various $m_{\rho}$. Comparison made between plots on the right and plots on the left for two cases, when $\Omega_{\chi}=\Omega_{\mathrm{DM}}$ or $\Omega_{\chi}=0.1 \Omega_{\mathrm{DM}}$. The horizontal shaded area is excluded because it violates perturbativity. The shaded region on the left is excluded by the invisible Higgs decay width measurements. 
Finally, we point out that there are regions in the model parameter space compatible with all the constraints mentioned above which can be reached by future LHC searches.

\section{Acknowledgments}

We are very grateful to Lorenzo Basso for helpful discussions. We would like to thank Hossein Ghorbani which was involved in the early stages of this research.

\section{References}

[1] Planck Collaboration, P. A. R. Ade et al., "Planck 2013 results. XVI. Cosmological parameters," Astron. Astrophys. 571 (2014) A16, arXiv:1303.5076 [astro-ph.C0].

[2] WMAP Collaboration, G. Hinshaw et al., "Nine-year wilkinson microwave anisotropy probe (wmap) observations: Cosmological parameter results," Astrophys.J.Suppl. 208 (2013) 19, arXiv:1212.5226 [astro-ph].

[3] R. J. Scherrer and M. S. Turner, "On the Relic, Cosmic Abundance of Stable Weakly Interacting Massive Particles," Phys. Rev. D33 (1986) 1585. [Erratum: Phys. Rev.D34,3263(1986)].

[4] G. Arcadi, M. Dutra, P. Ghosh, M. Lindner, Y. Mambrini, M. Pierre, S. Profumo, and F. S. Queiroz, "The Waning of the WIMP? A Review of Models, Searches, and Constraints," arXiv:1703.07364 [hep-ph].

[5] LUX Collaboration, D. S. Akerib et al., "First results from the LUX dark matter experiment at the Sanford Underground Research Facility," Phys. Rev. Lett. 112 (2014) 091303, arXiv:1310.8214 [astro-ph.CO].

[6] XENON100 Collaboration, E. Aprile et al., "Dark Matter Results from 225 Live Days of XENON100 Data," Phys. Rev. Lett. 109 (2012) 181301, arXiv:1207.5988 [astro-ph.CO].

[7] ATLAS Collaboration, G. Aad et al., "Observation of a new particle in the search for the Standard Model Higgs boson with the ATLAS detector at the LHC," Phys. Lett. B716 (2012) 1-29, arXiv:1207.7214 [hep-ex]

[8] CMS Collaboration, S. Chatrchyan et al., "Observation of a new boson at a mass of $125 \mathrm{GeV}$ with the CMS experiment at the LHC," Phys. Lett. B716 (2012) 30-61, arXiv:1207.7235 [hep-ex]. 
[9] L. Basso, "Resonant mono Higgs at the LHC," JHEP 04 (2016) 087, arXiv:1512.06381 [hep-ph].

[10] J. M. No, "Looking through the pseudoscalar portal into dark matter: Novel mono-Higgs and mono-Z signatures at the LHC," Phys. Rev. D93 no. 3, (2016) 031701, arXiv:1509.01110 [hep-ph].

[11] A. A. Petrov and W. Shepherd, "Searching for dark matter at LHC with Mono-Higgs production," Phys. Lett. B730 (2014) 178-183, arXiv:1311.1511 [hep-ph].

[12] A. Berlin, T. Lin, and L.-T. Wang, "Mono-Higgs Detection of Dark Matter at the LHC," JHEP 06 (2014) 078, arXiv:1402.7074 [hep-ph].

[13] O. Mattelaer and E. Vryonidou, "Dark matter production through loop-induced processes at the LHC: the s-channel mediator case," Eur. Phys. J. C75 no. 9, (2015) 436, arXiv:1508.00564 [hep-ph].

[14] L. Carpenter, A. DiFranzo, M. Mulhearn, C. Shimmin, S. Tulin, and D. Whiteson, "Mono-Higgs-boson: A new collider probe of dark matter," Phys. Rev. D89 no. 7, (2014) 075017, arXiv:1312.2592 [hep-ph].

[15] ATLAS Collaboration, M. Aaboud et al., "Search for Dark Matter Produced in Association with a Higgs Boson Decaying to $b \bar{b}$ using $36 \mathrm{fb}^{-1}$ of $p p$ collisions at $\sqrt{s}=13 \mathrm{TeV}$ with the ATLAS Detector," arXiv:1707.01302 [hep-ex].

[16] ATLAS Collaboration, M. Aaboud et al., "Search for dark matter in association with a Higgs boson decaying to two photons at $\sqrt{s}=13 \mathrm{TeV}$ with the ATLAS detector," arXiv:1706.03948 [hep-ex].

[17] CMS Collaboration, V. Khachatryan et al., "Search for dark matter, extra dimensions, and unparticles in monojet events in protonproton collisions at $\sqrt{s}=8$ TeV," Eur. Phys. J. C75 no. 5, (2015) 235, arXiv:1408.3583 [hep-ex].

[18] ATLAS Collaboration, G. Aad et al., "Search for new phenomena in final states with an energetic jet and large missing transverse momentum in pp collisions at $\sqrt{s}=8 \mathrm{TeV}$ with the ATLAS detector," Eur. Phys. J. C75 no. 7, (2015) 299, arXiv:1502.01518 [hep-ex]. [Erratum: Eur. Phys. J.C75,no.9,408(2015)].

[19] ATLAS Collaboration, G. Aad et al., "Search for dark matter in events with heavy quarks and missing transverse momentum in $p p$ collisions with the ATLAS detector," Eur. Phys. J. C75 no. 2, (2015) 92, arXiv:1410.4031 [hep-ex]. 
[20] M. Beltran, D. Hooper, E. W. Kolb, Z. A. C. Krusberg, and T. M. P. Tait, "Maverick dark matter at colliders," JHEP 09 (2010) 037, arXiv:1002.4137 [hep-ph].

[21] J. Goodman, M. Ibe, A. Rajaraman, W. Shepherd, T. M. P. Tait, and H.-B. Yu, "Constraints on Dark Matter from Colliders," Phys. Rev. D82 (2010) 116010, arXiv:1008.1783 [hep-ph].

[22] A. Rajaraman, W. Shepherd, T. M. P. Tait, and A. M. Wijangco, "LHC Bounds on Interactions of Dark Matter," Phys. Rev. D84 (2011) 095013, arXiv:1108.1196 [hep-ph].

[23] T. Lin, E. W. Kolb, and L.-T. Wang, "Probing dark matter couplings to top and bottom quarks at the LHC," Phys. Rev. D88 no. 6, (2013) 063510, arXiv:1303.6638 [hep-ph].

[24] J. Andrea, B. Fuks, and F. Maltoni, "Monotops at the LHC," Phys. Rev. D84 (2011) 074025, arXiv:1106.6199 [hep-ph].

[25] J.-L. Agram, J. Andrea, M. Buttignol, E. Conte, and B. Fuks, "Monotop phenomenology at the Large Hadron Collider," Phys. Rev. D89 no. 1, (2014) 014028, arXiv:1311.6478 [hep-ph].

[26] I. Boucheneb, G. Cacciapaglia, A. Deandrea, and B. Fuks, "Revisiting monotop production at the LHC," JHEP 01 (2015) 017, arXiv:1407.7529 [hep-ph].

[27] ATLAS Collaboration, G. Aad et al., "Search for dark matter in events with a hadronically decaying $\mathrm{W}$ or $\mathrm{Z}$ boson and missing transverse momentum in $p p$ collisions at $\sqrt{s}=8 \mathrm{TeV}$ with the ATLAS detector," Phys. Rev. Lett. 112 no. 4, (2014) 041802, arXiv:1309.4017 [hep-ex].

[28] ATLAS Collaboration, G. Aad et al., "Search for new particles in events with one lepton and missing transverse momentum in $p p$ collisions at $\sqrt{s}=8 \mathrm{TeV}$ with the ATLAS detector," JHEP 09 (2014) 037, arXiv:1407.7494 [hep-ex].

[29] CMS Collaboration, V. Khachatryan et al., "Search for physics beyond the standard model in final states with a lepton and missing transverse energy in proton-proton collisions at sqrt(s) $=8 \mathrm{TeV}$," Phys. Rev. D91 no. 9, (2015) 092005 , arXiv:1408.2745 [hep-ex].

[30] ATLAS Collaboration, G. Aad et al., "Search for dark matter in events with a Z boson and missing transverse momentum in pp collisions at $\sqrt{s}=8 \mathrm{TeV}$ with the ATLAS detector," Phys. Rev. D90 no. 1, (2014) 012004, arXiv:1404.0051 [hep-ex]. 
[31] N. F. Bell, J. B. Dent, A. J. Galea, T. D. Jacques, L. M. Krauss, and T. J. Weiler, "Searching for Dark Matter at the LHC with a Mono-Z," Phys. Rev. D86 (2012) 096011, arXiv:1209.0231 [hep-ph].

[32] Y. Bai and T. M. P. Tait, "Searches with Mono-Leptons," Phys. Lett. B723 (2013) 384-387, arXiv:1208.4361 [hep-ph].

[33] A. Alves and K. Sinha, "Searches for Dark Matter at the LHC: A Multivariate Analysis in the Mono- $Z$ Channel," Phys. Rev. D92 no. 11, (2015) 115013, arXiv:1507.08294 [hep-ph].

[34] U. Haisch, F. Kahlhoefer, and T. M. P. Tait, "On Mono-W Signatures in Spin-1 Simplified Models," Phys. Lett. B760 (2016) 207-213, arXiv: 1603.01267 [hep-ph].

[35] N. F. Bell, Y. Cai, J. B. Dent, R. K. Leane, and T. J. Weiler, "Dark matter at the LHC: Effective field theories and gauge invariance," Phys. Rev. D92 no. 5, (2015) 053008, arXiv:1503.07874 [hep-ph].

[36] N. F. Bell, Y. Cai, and R. K. Leane, "Mono-W Dark Matter Signals at the LHC: Simplified Model Analysis," JCAP 1601 no. 01, (2016) 051, arXiv:1512.00476 [hep-ph].

[37] CMS Collaboration, V. Khachatryan et al., "Search for new phenomena in monophoton final states in proton-proton collisions at $\sqrt{s}=8 \mathrm{TeV}$," Phys. Lett. B755 (2016) 102-124, arXiv:1410.8812 [hep-ex].

[38] ATLAS Collaboration, G. Aad et al., "Search for new phenomena in events with a photon and missing transverse momentum in $p p$ collisions at $\sqrt{s}=8 \mathrm{TeV}$ with the ATLAS detector," Phys. Rev. D91 no. 1, (2015) 012008, arXiv:1411.1559 [hep-ex]. [Erratum: Phys. Rev.D92,no.5,059903(2015)].

[39] P. J. Fox, R. Harnik, J. Kopp, and Y. Tsai, "Missing Energy Signatures of Dark Matter at the LHC," Phys. Rev. D85 (2012) 056011, arXiv:1109.4398 [hep-ph].

[40] W. Abdallah, J. Fiaschi, S. Khalil, and S. Moretti, "Mono-jet, -photon and -Z signals of a supersymmetric (B L) model at the Large Hadron Collider," JHEP 02 (2016) 157, arXiv: 1510.06475 [hep-ph].

[41] M. R. Buckley, D. Feld, and D. Goncalves, "Scalar Simplified Models for Dark Matter," Phys. Rev. D91 (2015) 015017, arXiv:1410.6497 [hep-ph].

[42] M. R. Buckley and D. Goncalves, "Constraining the Strength and CP Structure of Dark Production at the LHC: the Associated Top-Pair Channel," Phys. Rev. D93 no. 3, (2016) 034003, arXiv:1511.06451 [hep-ph]. [Phys. Rev.D93,034003(2016)]. 
[43] O. Buchmueller, S. A. Malik, C. McCabe, and B. Penning, "Constraining Dark Matter Interactions with Pseudoscalar and Scalar Mediators Using Collider Searches for Multijets plus Missing Transverse Energy," Phys. Rev. Lett. 115 no. 18, (2015) 181802, arXiv:1505.07826 [hep-ph].

[44] A. Berlin, S. Gori, T. Lin, and L.-T. Wang, "Pseudoscalar Portal Dark Matter," Phys. Rev. D92 (2015) 015005, arXiv: 1502.06000 [hep-ph].

[45] J. Kozaczuk and T. A. W. Martin, "Extending LHC Coverage to Light Pseudoscalar Mediators and Coy Dark Sectors," JHEP 04 (2015) 046. arXiv:1501.07275 [hep-ph].

[46] S. Esch, M. Klasen, and C. E. Yaguna, "Detection prospects of singlet fermionic dark matter," Phys. Rev. D88 (2013) 075017, arXiv:1308.0951 [hep-ph].

[47] L. Lopez-Honorez, T. Schwetz, and J. Zupan, "Higgs portal, fermionic dark matter, and a Standard Model like Higgs at 125 GeV," Phys. Lett. B716 (2012) 179-185, arXiv:1203.2064 [hep-ph].

[48] M. Pospelov and A. Ritz, "Higgs decays to dark matter: beyond the minimal model," Phys. Rev. D84 (2011) 113001, arXiv:1109.4872 [hep-ph].

[49] A. Freitas, S. Westhoff, and J. Zupan, "Integrating in the Higgs Portal to Fermion Dark Matter," JHEP 09 (2015) 015, arXiv:1506.04149 [hep-ph].

[50] K. Ghorbani, "Fermionic dark matter with pseudo-scalar Yukawa interaction," JCAP 1501 (2015) 015, arXiv:1408.4929 [hep-ph].

[51] J. R. Ellis, M. K. Gaillard, and D. V. Nanopoulos, "A Phenomenological Profile of the Higgs Boson," Nucl. Phys. B106 (1976) 292.

[52] B. A. Kniehl and M. Spira, "Low-energy theorems in Higgs physics," Z. Phys. C69 (1995) 77-88, arXiv:hep-ph/9505225 [hep-ph].

[53] CMS Collaboration, S. Chatrchyan et al., "Search for invisible decays of Higgs bosons in the vector boson fusion and associated ZH production modes," Eur. Phys. J. C74 (2014) 2980, arXiv:1404.1344 [hep-ex].

[54] CMS Collaboration, V. Khachatryan et al., "Searches for invisible decays of the Higgs boson in pp collisions at sqrt(s) = 7, 8, and $13 \mathrm{TeV}$," JHEP 02 (2017) 135, arXiv: 1610.09218 [hep-ex].

[55] A. Denner, S. Heinemeyer, I. Puljak, D. Rebuzzi, and M. Spira, "Standard Model Higgs-Boson Branching Ratios with Uncertainties," Eur. Phys. J. C71 (2011) 1753, arXiv:1107.5909 [hep-ph]. 
[56] ATLAS, CMS Collaboration, G. Aad et al., "Measurements of the Higgs boson production and decay rates and constraints on its couplings from a combined ATLAS and CMS analysis of the LHC $p p$ collision data at $\sqrt{s}=7$ and $8 \mathrm{TeV}$," arXiv: 1606.02266 [hep-ex].

[57] V. Barger, P. Langacker, M. McCaskey, M. J. Ramsey-Musolf, and G. Shaughnessy, "LHC Phenomenology of an Extended Standard Model with a Real Scalar Singlet," Phys. Rev. D77 (2008) 035005, arXiv:0706.4311 [hep-ph].

[58] Particle Data Group Collaboration, K. A. Olive et al., "Review of Particle Physics," Chin. Phys. C38 (2014) 090001.

[59] G. Belanger, F. Boudjema, A. Pukhov, and A. Semenov, "micrOMEGAs 3: A program for calculating dark matter observables," Comput. Phys. Commun. 185 (2014) 960-985, arXiv:1305.0237 [hep-ph].

[60] A. Belyaev, N. D. Christensen, and A. Pukhov, "CalcHEP 3.4 for collider physics within and beyond the Standard Model," Comput. Phys. Commun. 184 (2013) 1729-1769, arXiv:1207.6082 [hep-ph].

[61] J. Alwall, R. Frederix, S. Frixione, V. Hirschi, F. Maltoni, O. Mattelaer, H. S. Shao, T. Stelzer, P. Torrielli, and M. Zaro, "The automated computation of tree-level and next-to-leading order differential cross sections, and their matching to parton shower simulations," JHEP 07 (2014) 079, arXiv:1405.0301 [hep-ph].

[62] V. Hirschi and O. Mattelaer, "Automated event generation for loop-induced processes," JHEP 10 (2015) 146, arXiv:1507.00020 [hep-ph].

[63] J. Pumplin, D. R. Stump, J. Huston, H. L. Lai, P. M. Nadolsky, and W. K. Tung, "New generation of parton distributions with uncertainties from global QCD analysis," JHEP 07 (2002) 012, arXiv:hep-ph/0201195 [hep-ph].

[64] T. Sjostrand, S. Mrenna, and P. Z. Skands, "PYTHIA 6.4 Physics and Manual," JHEP 05 (2006) 026, arXiv: hep-ph/0603175 [hep-ph].

[65] DELPHES 3 Collaboration, J. de Favereau, C. Delaere, P. Demin, A. Giammanco, V. Lematre, A. Mertens, and M. Selvaggi, "DELPHES 3, A modular framework for fast simulation of a generic collider experiment," JHEP 02 (2014) 057, arXiv:1307.6346 [hep-ex].

[66] M. Cacciari, G. P. Salam, and G. Soyez, "FastJet User Manual," Eur. Phys. J. C72 (2012) 1896, arXiv:1111.6097 [hep-ph].

[67] M. Cacciari, G. P. Salam, and G. Soyez, "The Anti-k(t) jet clustering algorithm," JHEP 04 (2008) 063, arXiv:0802.1189 [hep-ph]. 
[68] E. Conte, B. Fuks, and G. Serret, "MadAnalysis 5, A User-Friendly Framework for Collider Phenomenology," Comput. Phys. Commun. 184 (2013) 222-256, arXiv:1206.1599 [hep-ph].

[69] B. Dumont, B. Fuks, S. Kraml, S. Bein, G. Chalons, E. Conte, S. Kulkarni, D. Sengupta, and C. Wymant, "Toward a public analysis database for LHC new physics searches using MADANALYSIS 5," Eur. Phys. J. C75 no. 2, (2015) 56, arXiv: 1407.3278 [hep-ph].

[70] J. M. Campbell, H. B. Hartanto, and C. Williams, "Next-to-leading order predictions for $Z \gamma+$ jet and Z $\gamma \gamma$ final states at the LHC," JHEP 11 (2012) 162 , arXiv:1208.0566 [hep-ph].

[71] F. Maltoni, K. Mawatari, and M. Zaro, "Higgs characterisation via vector-boson fusion and associated production: NLO and parton-shower effects," Eur. Phys. J. C74 no. 1, (2014) 2710, arXiv:1311.1829 [hep-ph].

[72] LHC Higgs Cross Section Working Group Collaboration, J. R. Andersen et al., "Handbook of LHC Higgs Cross Sections: 3. Higgs Properties," arXiv:1307.1347 [hep-ph].

[73] ATLAS Collaboration, G. Aad et al., "Measurement of the isolated di-photon cross-section in $p p$ collisions at $\sqrt{s}=7 \mathrm{TeV}$ with the ATLAS detector," Phys. Rev. D85 (2012) 012003, arXiv:1107.0581 [hep-ex].

[74] F. Febres Cordero, L. Reina, and D. Wackeroth, "W- and Z-boson production with a massive bottom-quark pair at the Large Hadron Collider," Phys. Rev. D80 (2009) 034015, arXiv:0906.1923 [hep-ph].

[75] ATLAS Collaboration, T. A. collaboration, "Study of the Higgs boson properties and search for high-mass scalar resonances in the $H \rightarrow Z Z^{*} \rightarrow 4 \ell$ decay channel at $\sqrt{s}=13 \mathrm{TeV}$ with the ATLAS detector,".

[76] ATLAS Collaboration, T. A. collaboration, "Searches for heavy ZZ and ZW resonances in the llqq and vvqq final states in pp collisions at sqrt(s) $=13 \mathrm{TeV}$ with the ATLAS detector,".

[77] ATLAS Collaboration, T. A. collaboration, "Search for a high-mass Higgs boson decaying to a pair of $W$ bosons in $p p$ collisions at $\sqrt{s}=13 \mathrm{TeV}$ with the ATLAS detector,".

[78] ATLAS Collaboration, T. A. collaboration, "Search for pair production of Higgs bosons in the $b \bar{b} b \bar{b}$ final state using proton-proton collisions at $\sqrt{s}=13 \mathrm{TeV}$ with the ATLAS detector,". 
[79] CMS Collaboration, C. Collaboration, "Search for resonant Higgs boson pair production in the $\mathrm{b} \overline{\mathrm{b}} \tau^{+} \tau^{-}$final state using 2016 data,".

[80] S. Baek, P. Ko, and J. Li, "Minimal renormalizable simplified dark matter model with a pseudoscalar mediator," Phys. Rev. D95 no. 7, (2017) 075011, arXiv: 1701.04131 [hep-ph]. 